\title{
A COMPARATIVE STUDY OF CLONIDINE AND DEXMEDETOMIDINE AS ADJUVANT IN NEURAXIAL BLOCK - A CASE SERIES
}

Anaesthesiology

Dr Joshi Nirali K. Tutor, Department OfAnaesthesia, B. J. M. C. Ahmedabad.

Dr Parasmani*

Senior Resident, Department Of Anaesthesia, V. M. M. C. And Safdarjung Hospital, New

Dr Mukesh I.

Shukla Delhi. *Corresponding Author

Associate Professor, Department OfAnaesthesia, B. J. M. C. Ahmedabad.

\section{ABSTRACT}

BACKGROUND-Most of the lower abdominal surgeries are performed under spinal anaesthesia which is a popular technique using hyperbaric local anaesthetic solutions such as $0.5 \%$ Bupivacaine. The advantages are simplicity of technique, rapid onset of action and reliability in producing uniform sensory and motor blockade. Main disadvantage of using plain local anaesthetic agent arelimited duration of action and lack of longer postoperative analgesia. To overcome this problem, administration of different adjuvant in local anaesthetic is an excellent technique.

AIMS-To compare the effect of adding Clonidine and Dexmedetomidine to Bupivacaine for neuraxial block.

METHODS-This study was be conducted after the approval of institutional ethical committee. It is a prospective study in which 75 selected patients who were posted for lower abdominal surgeries were randomly allotted into three groups. Group B-Inj. $0.5 \%$ Heavy Bupivacaine $3.2 \mathrm{cc}(16$ $\mathrm{mg})+$ Inj. Normal saline Group C -Inj. $0.5 \%$ Heavy Bupivacaine 3.2cc(16 mg) + Inj.Clonidine $30 \mathrm{mcg}$ Group D -Inj.0.5\% Heavy Bupivacaine $3.2 \mathrm{cc}(16 \mathrm{mg})+$ Inj.Dexmedetomidine $3 \mathrm{mcg}$, Total volume injected in all group was $3.5 \mathrm{ml}$. The end of drug injection was taken as zero time. Onset, duration of sensory blockade, duration of motor blockade was noted.

RESULTS - prolonged sensory and motor blockage and superior post-operative analgesia was observed in group D.

CONCLUSION-Addition of Dexmeditomidine $3 \mathrm{mcg}$ is significantly more effective than plain $0.5 \%$ Bupivacaine or when Clonidine $30 \mathrm{mcg}$ was used as adjuvant, for prolongation of sensory and motor blockage and post-operative analgesia.

\section{KEYWORDS}

Dexmediotomidine, Clonidine, Intrathecal.

\section{INTRODUCTION:}

Most of the lower abdominal surgeries are performed under spinal anaesthesia which is a popular technique using hyperbaric local anaesthetic solutions such as $0.5 \%$ Bupivacaine. The advantages are simplicity of technique, rapid onset of action and reliability in producing uniform sensory and motor blockade. Main disadvantage of using plain local anaesthetic agent are limited duration of action and lack of longer postoperative analgesia. To overcome this problem, administration of different adjuvant in local anaesthetic is an excellent technique.Advantages of adjuvents areincreased quality of sensory and motor blockade, increased duration of block and decreased postoperative pain.It acts as synergistic to local anaesthetics which lower local anaesthetic requirement, decreases side effects. Various drugs like opioids, neostigmine, midazolam, preservative free ketamine,clonidine,dexmedetomidine etc. are used as adjuvants intrathecally in clinical practice. Dexmedetomidine and clonidine are selective $\alpha 2$ adrenergic agonist. Small doses of selective $\alpha 2$ adrenergic agonist used in combination with bupivacaine in neuroaxial block produce a prolongation in the duration of motor and sensory block with nonsignificant hemodynamic changes.

\section{AIMS AND OBJECTIVES:}

This study was done to compare the effect of adding Clonidine and Dexmedetomidine to Bupivacaine for neuraxial blockade and compare the onset and duration of sensory and motor block, analgesiaand side effects.

\section{MATERIALS AND METHODS:}

Institutional ethical approval was obtained.Total 75 patients undergoing lower abdominal surgeries were selected.Adult patientsup to 55 yearsboth genders,ASA status I and II, body Weight 40 to $70 \mathrm{kgs}$ and height 150 to 170 cmwith normal coagulation profile were included in the study. Patients with ASA status III, IV, V, paediatric and geriatric patients, patients with spine abnormalities or psychiatric disorder, patients on anti-hypertensive and anticoagulant medications and those with history of aallergy or coagulopathy were excluded from the study. This was a prospective study in which 75 selected patients who were posted for lower abdominal surgeries are randomly allotted into three groups according to inclusion criteria.

- Group B -Inj. $0.5 \%$ Heavy Bupivacaine 3.2cc(16 mg) + Inj. Normal saline

- Group C -Inj.0.5\% Heavy Bupivacaine 3.2cc(16 mg) + Inj.Clonidine $30 \mathrm{mcg}$
- Group D -Inj.0.5\% Heavy Bupivacaine 3.2cc(16 mg) + Inj.Dexmedetomidine $3 \mathrm{mcg}$

By adding $0.9 \%$ normal saline to local anaethesticagent's total volume injected in all group is $3.5 \mathrm{ml}$.Study drug was injected after bevel directed upward and checking for free flow of CSF over 10-15 seconds.After that patients were immediately changed to the supine position. The end of drug injection was taken as zero time. Onset time for sensory and motor blockage was noted.A sensory level of T6 was considered adequate to allow surgery to proceed.The duration of sensory blockade (time to regression of sensory T6 to S1) was recorded.The duration of motor blockade (time to achieve Modified Bromage scale 3 to 0)was noted.Intraoperative Complication like hypotension, bradycardia, nausea, vomiting, sedation, respiratory depression can occur and treated accordingly. Intra-op blood loss is replaced as indicated. No additional sedative medications are given during the surgery.

\section{OBSERVATIONS:} DEMOGRAPHIC DATA:

There was no statistical difference between the three groups.

\section{Demographic data}

\begin{tabular}{|c|c|c|c|c|}
\hline & & Group B & Group C & Group D \\
\hline \multicolumn{2}{|c|}{\begin{tabular}{|l} 
Age(years) \\
\end{tabular}} & $35.90 \pm 8.49$ & $39.80 \pm 8.44$ & $33.95 \pm 12.62$ \\
\hline \multicolumn{2}{|c|}{ Weight(kg) } & $57.30 \pm 8.18$ & $\mid 56.85 \pm 9.64$ & $55.90 \pm 8.06$ \\
\hline \multicolumn{2}{|c|}{ Height(cm) } & $157.20 \pm 4.91$ & $159.95 \pm 5.62$ & $159.15 \pm 6.70$ \\
\hline \multirow[t]{2}{*}{ Gender } & Male & 8 & 15 & 16 \\
\hline & Female & 12 & 5 & 4 \\
\hline
\end{tabular}

Gender

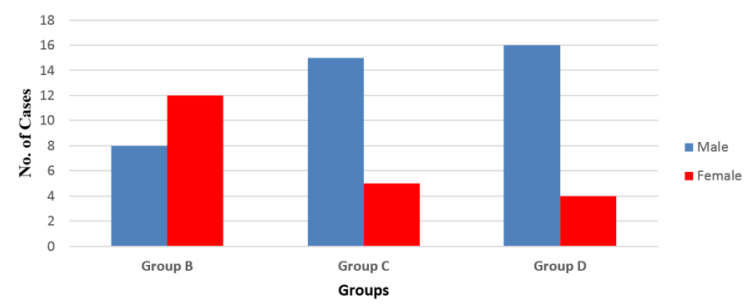




ASA risk status
\begin{tabular}{|l|l|l|l|}
\hline & Group B & Group C & Group D \\
\hline ASA risk I & 13 & 7 & 9 \\
\hline ASA risk II & 7 & 13 & 11 \\
\hline
\end{tabular}

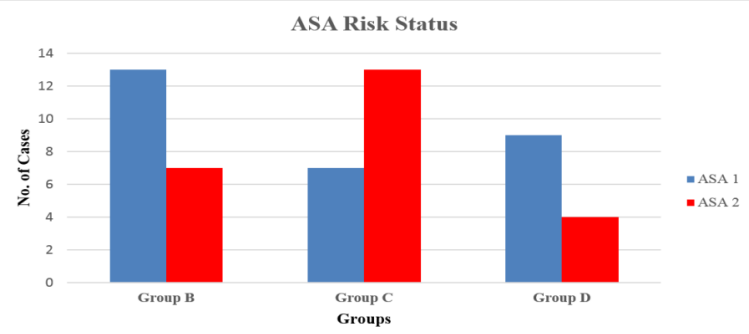

Total duration of surgery

\begin{tabular}{|l|l|l|l|}
\hline & Group B & Group C & Group D \\
\hline $\begin{array}{l}\text { Duration of surgery } \\
\text { (min) }\end{array}$ & $78.25 \pm 36.32$ & $96.55 \pm 36.52$ & $83.85 \pm 28.98$ \\
\hline
\end{tabular}

Time of onset of Sensory blockade

\begin{tabular}{|l|l|l|l|}
\hline & Group B & Group C & Group D \\
\hline $\begin{array}{l}\text { Time to reach sensory } \\
\text { level T12 (min) }\end{array}$ & $2.20 \pm 0.89$ & $1.75 \pm 0.72$ & $2.10 \pm 0.64$ \\
\hline $\begin{array}{l}\text { Time to reach sensory } \\
\text { level T10 (min) }\end{array}$ & $4.35 \pm 0,75$ & $3.40 \pm 1.05$ & $4.25 \pm 1.77$ \\
\hline $\begin{array}{l}\text { Time to reach sensory } \\
\text { level T6 (min) }\end{array}$ & $9.95 \pm 3.63$ & $8.35 \pm 2.72$ & $10.10 \pm 3.96$ \\
\hline
\end{tabular}

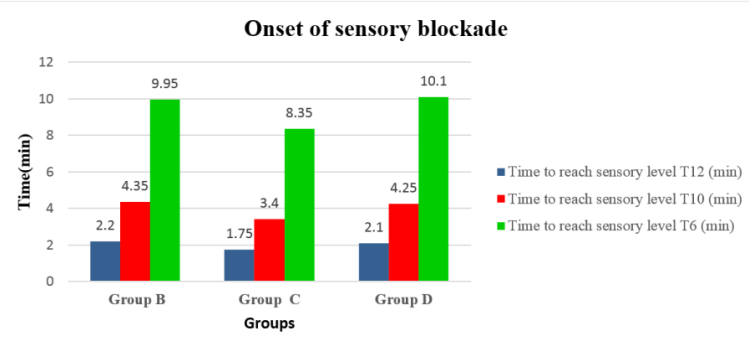

Duration of sensory block

(Time of regression of sensory S1 from T6 level)

\begin{tabular}{|l|l|l|l|}
\hline & Group B & Group C & Group D \\
\hline $\begin{array}{l}\text { Duration of sensory } \\
\text { block (min) }\end{array}$ & $314.00 \pm 24.96$ & $504.505 \pm 42.91$ & $576.35 \pm 95.00$ \\
\hline
\end{tabular}

block (min)

Onset of motor blockade

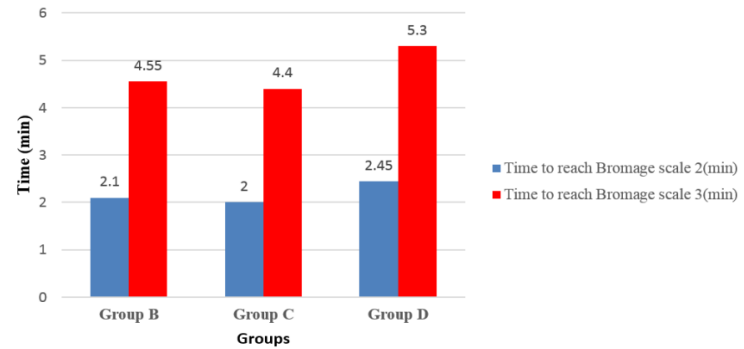

Duration of Motor Block

(Time to achieve Modified Bromage scale 0 to 3)

\begin{tabular}{|l|l|l|l|}
\hline & Group B & Group C & Group D \\
\hline $\begin{array}{l}\text { Duration of motor } \\
\text { block (min) }\end{array}$ & $237.40 \pm 21.12$ & $426.50 \pm 40.33$ & $495.45 \pm 93.20$ \\
\hline
\end{tabular}

Two segment regression time

(Time for regression of two segments from $\mathrm{T} 6$ level)

\begin{tabular}{|l|l|l|l|}
\hline & Group B & Group C & Group D \\
\hline $\begin{array}{l}\text { Time of two segment } \\
\text { regression (min) }\end{array}$ & $60.50 \pm 7.42$ & $107.50 \pm 28.47$ & $114.90 \pm 41.97$ \\
\hline
\end{tabular}

Duration of effective Analgesia

(Time of giving spinal anaesthesia till [VAS] visual analogue scale $>3$ )

\begin{tabular}{|l|l|l|l|}
\hline & Group B & Group C & Group D \\
\hline $\begin{array}{l}\text { Duration of effective } \\
\text { analgesia(min) }\end{array}$ & $202.80 \pm 21.30$ & $366.85 \pm 48.76$ & $359.65 \pm 62.51$ \\
\hline
\end{tabular}

COMPARISON OF DURATION OF SENSORY AND MOTORBLOCKADE:

\begin{tabular}{|l|l|l|l|}
\hline & GROUP B & GROUP C & GROUP D \\
\hline $\begin{array}{l}\text { Time to reach sensory } \\
\text { T10(min) }\end{array}$ & $4.35 \pm 0.75$ & $3.40 \pm 1.05$ & $4.25 \pm 1.77$ \\
\hline $\begin{array}{l}\text { Time to reach sensory } \\
\text { T6(min) }\end{array}$ & $9.95 \pm 3.63$ & $8.35 \pm 2.72$ & $10.10 \pm 3.96$ \\
\hline $\begin{array}{l}\text { Time to reach M } \\
\text { Bromage 2(min) }\end{array}$ & $2.10 \pm 0.91$ & $2.00 \pm 0.79$ & $2.45 \pm 1.15$ \\
$\begin{array}{l}\text { Time to reach M } \\
\text { Bromage 3(min) }\end{array}$ & $4.55 \pm 1.76$ & $4.40 \pm 1.57$ & $5.30 \pm 2.68$ \\
\hline $\begin{array}{l}\text { Duration of motor } \\
\text { block(min) }\end{array}$ & $237.40 \pm 21.12$ & $426.50 \pm 40.33$ & $495.45 \pm 93.20$ \\
\hline $\begin{array}{l}\text { Duration of effective } \\
\text { analgesia(min) }\end{array}$ & $202.80 \pm 21.30$ & $366.85 \pm 48.76$ & $359.65 \pm 62.51$ \\
\hline
\end{tabular}

PERI-OPERATIVE PULSE RATE:The difference in mean pulse rate between three groups were statistically not significant.

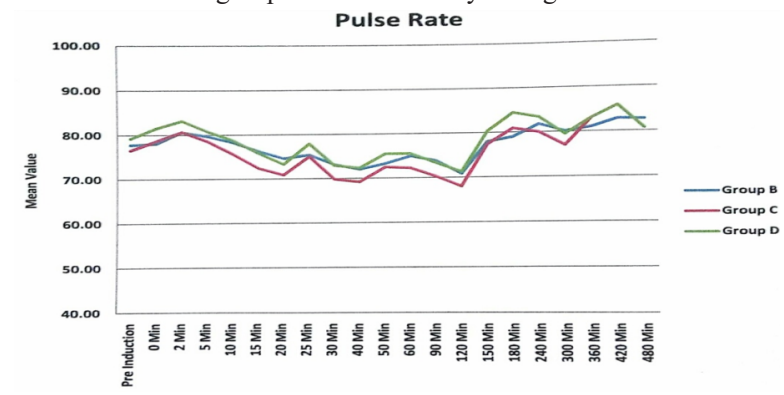

PERIOPERATIVE SYSTOLIC BLOOD PRESSURE:The difference in mean pulse rate between three groups were statistically not significant.

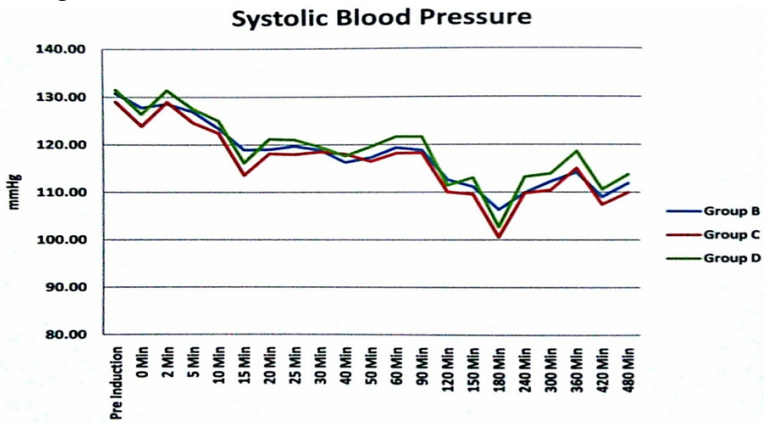

PERIOPERATIVE DIASTOLIC BP: The difference in mean pulse rate between three groups were statistically not significant.

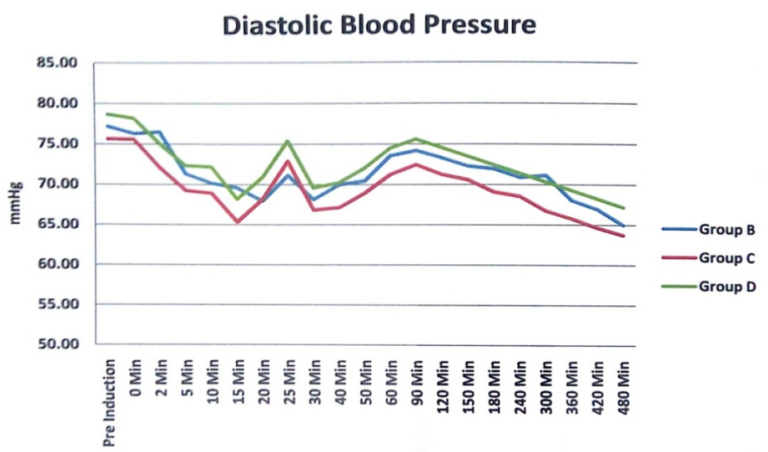

PERIOPERATIVE SPO2: The difference in mean BP between three groups were statistically not significant.

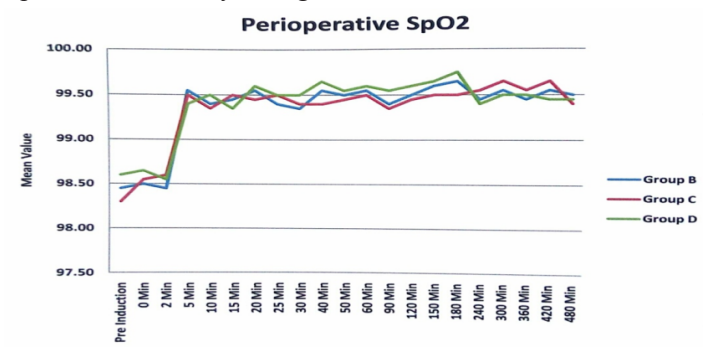




\section{PERIOPERATIVE COMPLICATIONS}

\begin{tabular}{|c|c|c|c|}
\hline Complications & Group B & Group C & Group D \\
\hline Bradycardia & 0 & 2 & 0 \\
\hline Hypotension & 7 & 8 & 7 \\
\hline Nausea & 0 & 0 & 0 \\
\hline Vomiting & 2 & 2 & 2 \\
\hline Pruritus & 0 & 0 & 0 \\
\hline Shivering & 0 & 0 & 0 \\
\hline Respiratory Depression & 0 & 0 & 0 \\
\hline
\end{tabular}

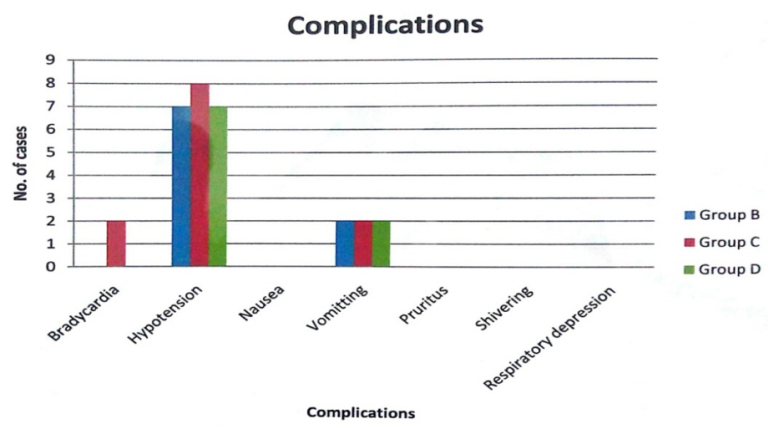

\section{DISCUSSION:}

\section{TIME OF ONSET OF SENSORY BLOCK}

According to V Mahendru et al, P Shethi et al study Time for onset of sensory levelT6 was shorter in bupivacaine plus clonidine group as compared to bupivacaine plus dexmeditimidine and bupivacaine only group. According to T Suryashree et al timefor onset of sensory level
T6 wa observed to be shorter in bupivacaine plus dexmeditimidine group as compared to bupivacaine plus clonidine.In our study time for onset of sensory levelT6 was observed to be shorter in bupivacaine plus clonidine group as compare to bupivacaine plus dexmeditimidine and bupivacaine only group

Time between the end of injection of test drug to achieve 16 sensory level

\begin{tabular}{|l|l|l|l|l|l|l|}
\hline No. & Author & Year & Bupivacaine & Adjuvant & Mean time of Onset of T6 Sensory block \\
\cline { 4 - 7 } & & & & Drug & Dose & \\
\hline $\mathbf{1 .}$ & V.Mahendru & 2013 & $12.5 \mathrm{mg}$ & & & $10.1 \pm 3.5$ \\
\hline & & & & Clonidine & $30 \mathrm{mcg}$ & $9.5 \pm 3$ \\
\hline & & & & cDexmedetomidine & $5 \mathrm{mcg}$ & $10.3 \pm 3.3$ \\
\hline $\mathbf{2 .}$ & P. Shethi & 2015 & $12.5 \mathrm{mg}$ & & & $16 \pm 3.85$ \\
\hline & & & & Clonidine & $30 \mathrm{mcg}$ & $14 \pm 4.11$ \\
\hline & & & & cDexmedetomidine & $3 \mathrm{mcg}$ & $17 \pm 4.51$ \\
\hline $\mathbf{3 .}$ & Suryasree & 2015 & $15 \mathrm{mg}$ & Clonidine & $30 \mathrm{mcg}$ & $4.3 \pm 1.12$ \\
\hline & & & & cDexmedetomidine & $5 \mathrm{mcg}$ & $4.03 \pm 1.0$ \\
\hline $\mathbf{4 .}$ & Our study & 2017 & $16 \mathrm{mg}$ & & & $9.95 \pm 3.63$ \\
\hline & & & & Clonidine & $30 \mathrm{mcg}$ & $8.35 \pm 2.72$ \\
\hline & & & & cDexmedetomidine & $3 \mathrm{mcg}$ & $10.10 \pm 3.96$ \\
\hline
\end{tabular}

\section{TIME OF ONSET OF MOTOR BLOCK}

According to G E Kanazi et al, V Mahendru et al, P Shethi et al. the time for onset of motor block was shorter in bupivacaine plus clonidine group and bupivacaine plus dexmeditimidine group as compared to bupivacaine only group.According to $\mathrm{T}$ Suryashree et. al. time for onset of motor block was found to be shorter in bupivacaine plus dexmeditimidine group as compare to bupivacaine plus clonidine.In our study time for onset of motor block was observed to be shorter in bupivacaine plus clonidine group as as compared to bupivacaine plus dexmeditimidine group and bupivacaine only group.

Onset of motor block:Time from the end of injection of test drug to achieve Modified Bromage scale 3

\begin{tabular}{|l|l|l|l|l|l|l|}
\hline No. & Author & Year & \multirow{2}{*}{ Bupivacaine } & Adjuvant & Mean time of onset of motor block \\
\cline { 5 - 6 } & & & & Drug & \\
\hline $\mathbf{1 .}$ & G.E.Kanazi & 2006 & $12 \mathrm{mg}$ & & & $20.7 \pm 10.3$ \\
\hline & & & & Clonidine & $30 \mathrm{mcg}$ & $11.7 \pm 5.9$ \\
\hline $\mathbf{2 .}$ & V. Mahendr & 2013 & 12.5 & & $3 \mathrm{mcg}$ & $13.2 \pm 5.6$ \\
\hline & & & & Clonidine & $30 \mathrm{mcg}$ & $9.2 \pm 2.9$ \\
\hline & & & & Dexmedetomidine & $5 \mathrm{mcg}$ & $9.8 \pm 3.6$ \\
\hline $\mathbf{3 .}$ & P. Shethi & 2015 & 12.5 & & & $9.7 \pm 3.2$ \\
\hline & & & & Clonidine & $30 \mathrm{mcg}$ & $15.0 \pm 3.4$ \\
\hline & & & & Dexmedetomidine & $3 \mathrm{mcg}$ & $9.0 \pm 1.8$ \\
\hline $\mathbf{4 .}$ & T. Suryasree & 2015 & 15 & Clonidine & $30 \mathrm{mcg}$ & $10.0 \pm 1.7$ \\
\hline & & & & Dexmedetomidine & $5 \mathrm{mcg}$ & $6.57 \pm 1.48$ \\
\hline $\mathbf{5 .}$ & Our Study & 2017 & 2017 & & & $5.27 \pm 1.25$ \\
\hline & & & & Clonidine & $30 \mathrm{mcg}$ & $4.55 \pm 1.76$ \\
\hline & & & & Dexmedetomidine & $3 \mathrm{mcg}$ & $5.30 \pm 2.68$ \\
\hline
\end{tabular}

\section{1) DURATION OF MOTOR BLOCKADE}

According to G E Kanazi et al, P Shethi et al, V Mahendru et.al. the time of Duration of motor blockage was significantly prolonged in in bupivacaine plus clonidine group and bupivacaine plus dexmeditimidine group as compared to bupivacaine only group. According to $\mathrm{T}$
Suryashree et. al. the time of duration of motor block was observed to be significantly prolonged in bupivacaine plus dexmeditimidine group as compared to bupivacaine plus clonidine.In our study the time of duration of motor blockade was prolonged in bupivacaine plus dexmeditimidine group as compared to bupivacaine plus clonidine. 
Duration of Motor Block

\begin{tabular}{|l|l|l|l|}
\hline No. & Author & Year & Bupivacaine \\
\hline $\mathbf{1 .}$ & G.E.Kanazi & 2006 & $12 \mathrm{mg}$ \\
\hline & & & \\
\hline & & & \\
\hline $\mathbf{2 .}$ & V. Mahendr & 2013 & 12.5 \\
\hline & & & \\
\hline $\mathbf{3 .}$ & & & \\
\hline & P. Shethi & 2015 & 12.5 \\
\hline & & & \\
\hline $\mathbf{4 .}$ & & & \\
\hline $\mathbf{5 .}$ & T. Suryasree & 2015 & 15 \\
\hline & & & \\
\hline & Our Study & 2017 & 2017 \\
\hline
\end{tabular}

\section{2) DURATION OF SENSORY BLOCKADE}

According to G E Kanazi et al, P Shethi et al. time of duration of sensory block was significantly prolonged in in bupivacaine plus clonidine group and bupivacaine plus dexmeditimidine group than bupivacaine only group.According to $\mathrm{T}$ Suryashree et al. time of

\begin{tabular}{|l|l|l|}
\hline \multicolumn{2}{|l|}{ Adjuvant } & \multirow{2}{*}{ Mean duration of motor block } \\
\cline { 1 - 3 } Drug & Dose & \\
\hline & & $163 \pm 47$ \\
\hline Clonidine & $30 \mathrm{mcg}$ & $216 \pm 35$ \\
\hline Dexmedetomidine & $3 \mathrm{mcg}$ & $250 \pm 76$ \\
\hline & & $161.5 \pm 19.8$ \\
\hline Clonidine & $30 \mathrm{mcg}$ & $198.7 \pm 26.4$ \\
\hline Dexmedetomidine & $5 \mathrm{mcg}$ & $273.3 \pm 24.6$ \\
\hline & & $175 \pm 28.8$ \\
\hline Clonidine & $30 \mathrm{mcg}$ & $229 \pm 42.57$ \\
\hline Dexmedetomidine & $3 \mathrm{mcg}$ & $253 \pm 38.4$ \\
\hline Clonidine & $30 \mathrm{mcg}$ & $223.03 \pm 45.35$ \\
\hline Dexmedetomidine & $5 \mathrm{mcg}$ & $269.6 \pm 45.05$ \\
\hline & & $237.40 \pm 21.12$ \\
\hline Clonidine & $30 \mathrm{mcg}$ & $426.50 \pm 40.33$ \\
\hline Dexmedetomidine & $3 \mathrm{mcg}$ & $495.45 \pm 93.2$ \\
\hline
\end{tabular}

duration of sensory block was significantly prolonged in in bupivacaine plus dexmeditimidine group as compare to bupivacaine plus clonidine.In our study the time of duration of sensory block was prolonged in in bupivacaine plus clonidine group and bupivacaine plus dexmeditimidine group as compared to bupivacaine only group.

Duration of Sensory block: Time to regression of sensory S1 from T6 level

\begin{tabular}{|l|l|l|l|l|l|l|}
\hline No. & Author & Year & Bupivacaine & \multicolumn{2}{l|}{ Adjuvant } & Mean duration of sensory block \\
\cline { 5 - 6 } & & & & Drug & \\
\hline $\mathbf{1 .}$ & G.E. Kanazi & 2006 & $12 \mathrm{mg}$ & & & $190 \pm 48$ \\
\hline & & & & Clonidine & $30 \mathrm{mcg}$ & $272 \pm 38$ \\
\hline & & & & Dexmedetomidine & $3 \mathrm{mcg}$ & $303 \pm 75$ \\
\hline $\mathbf{2 .}$ & P. Shethi & 2015 & 12.5 & & & $199.8 \pm 32.9$ \\
\hline & & & & Clonidine & $30 \mathrm{mcg}$ & $278.6 \pm 26.4$ \\
\hline & & & & cDexmedetomidine & $3 \mathrm{mcg}$ & $306.6 \pm 51$ \\
\hline $\mathbf{3 .}$ & T. Suryasree & 2015 & 12.5 & Clonidine & $30 \mathrm{mcg}$ & $300.83 \pm 35.06$ \\
\hline & & & & Dexmedetomidine & $5 \mathrm{mcg}$ & $345.93 \pm 45.9$ \\
\hline $\mathbf{4 .}$ & Our study & 2017 & $16 \mathrm{mg}$ & & & $314 \pm 24.96$ \\
\hline & & & & Clonidine & $30 \mathrm{mcg}$ & $504.50 \pm 42.91$ \\
\hline & & & & cDexmedetomidine & $5 \mathrm{mcg}$ & $576.35 \pm 95$ \\
\hline
\end{tabular}

\section{3) DURATION OF EFFECTIVE ANALGESIA}

According to V Mahendru et al, S L Solanki et al, P Shethi et al. time of duration of effective analgesia was longest in Bupivacaine plus Dexmeditomidine group but duration of effective analgesia was significantly prolonged in Bupivacaine plus Dexmeditomidine and Bupivacaine plus Clonidine group as compared to bupivacaine
group.According to T Suryashree et al, M Prabhakar et al duration of effective analgesia is significantly prolonged in Bupivacaine plus Dexmeditomidine group as compared to Bupivacaine plus Clonidine group. In our study duration of effective analgesia was significantly prolonged in Bupivacaine plus Dexmeditomidine and Bupivacaine plus Clonidine group as compared to bupivacaine only group.

\begin{tabular}{|c|c|c|c|c|c|}
\hline \multicolumn{6}{|c|}{ Doses of Dexmedefomidine and Cloridine } \\
\hline No. & Author & Year & Bupivacaine & Adjuvant & \\
\hline & & & & Drug & Dose \\
\hline \multirow[t]{2}{*}{1} & G.E.Kanazi & 2006 & $12 \mathrm{mg}$ & Clonidine & \\
\hline & & & & Dexmedetomidine & $5 \mathrm{mcg}$ \\
\hline \multirow[t]{2}{*}{2} & AI Mustafa & 2009 & $12.5 \mathrm{mg}$ & Dexmedetomidine & $5 \mathrm{mcg}$ \\
\hline & & & & & $10 \mathrm{mcg}$ \\
\hline 3 & R Gupta & 2011 & $12.5 \mathrm{mg}$ & Dexmedetomidine & $5 \mathrm{mcg}$ \\
\hline 4 & Mohamad AA & 2012 & $10 \mathrm{mg}$ & Dexmedetomidine & $5 \mathrm{mcg}$ \\
\hline \multirow[t]{2}{*}{5} & HALA E A EID & & $15 \mathrm{mg}$ & Dexmedetomidine & $10 \mathrm{mcg}$ \\
\hline & & & & & $15 \mathrm{mcg}$ \\
\hline \multirow[t]{2}{*}{6} & V.Mahendru & 2013 & $12.5 \mathrm{mg}$ & Clonidine & $30 \mathrm{mcg}$ \\
\hline & & & & Dexmedetomidine & $5 \mathrm{mcg}$ \\
\hline \multirow[t]{2}{*}{7} & S.L.Solanki & 2013 & $15 \mathrm{mg}$ & Clonidine & $50 \mathrm{mcg}$ \\
\hline & & & & Dexmedetomidine & $5 \mathrm{mcg}$ \\
\hline 8 & M.Gupta & 2014 & $15 \mathrm{mg}$ & Dexmedetomidine & $5 \mathrm{mcg}$ \\
\hline 9 & D.Shukla & & $15 \mathrm{mg}$ & Dexmedetomidine & $10 \mathrm{mcg}$ \\
\hline 10 & V. Chastrath & 2014 & $12.5 \mathrm{mg}$ & Dexmedetomidine & $10 \mathrm{mcg}$ \\
\hline 11 & Hem Anand Naragam & 2014 & $8 \mathrm{mg}$ & Dexmedetomidine & $5 \mathrm{mcg}$ \\
\hline \multirow[t]{2}{*}{12} & P.Shethi & 2015 & 12.5 & Clonidine & $30 \mathrm{mcg}$ \\
\hline & & & & Dexmedetomidine & $3 \mathrm{mcg}$ \\
\hline \multirow[t]{2}{*}{13} & T.Suryasree & 2015 & $15 \mathrm{mg}$ & Clonidine & $30 \mathrm{mcg}$ \\
\hline & & & & Dexmedetomidine & $5 \mathrm{mcg}$ \\
\hline 14 & Ch.Srinivas Rao & 2015 & $12.5 \mathrm{mg}$ & Dexmedetomidine & $5 \mathrm{mcg}$ \\
\hline \multirow[t]{2}{*}{15} & Murali Prabhakar & 2015 & $12.5 \mathrm{mg}$ & Clonidine & $50 \mathrm{mcg}$ \\
\hline & & & & Dexmedetomidine & $2 \mathrm{mcg}$ \\
\hline 16 & R.Tripathi & 2015 & $15 \mathrm{mg}$ & Dexmedetomidine & $5 \mathrm{mcg}$ \\
\hline \multirow[t]{2}{*}{17} & Our study & 2017 & $16 \mathrm{mg}$ & Clonidine & $30 \mathrm{mcg}$ \\
\hline & & & & Dexmedetomidine & $3 \mathrm{mcg}$ \\
\hline
\end{tabular}




\section{CONCLUSION:}

Effects of Dexmeditomidine $3 \mathrm{mcg}$ and Clonidine $30 \mathrm{mcg}$ is significantly more effective than $0.5 \%$ Bupivacaine only for prolongation of sensory and motor blockage and post-operative analgesia. Effect of Dexmeditomidine $3 \mathrm{mcg}$ is superior to Clonidine $30 \mathrm{mcg}$ for prolongation of duration of sensory and motor block and post-operative analgesia.

\section{REFERENCES:}

1) Mahendru V, Tewari A, Katyal S, Grewal A, Singh M R, Katyal R. A comparison of Mahendru V, Tewari A, Katyal S, Grewal A, Singh M R, Katyal R. A comparison of
intrathecal dexmedetomidine, clonidine, and fentanyl as adjuvants to hyperbaric bupivacaine for lower limb surgery: A double blind controlled study. J Anaesthesiol Clin Pharmacol2013;29:496-502

2) Sethi P1, Sindhi S2, Verma A2, Tulsiani KL2 Dexmedetomidine versus propofol in dilatation and curettage: An open-label pilot randomized controlled trial. Saudi J Anaesth. 2015 Jul-Sep;9(3):258-62. doi: 10.4103/1658-354X.154699.

3) Solanki SL1, Bharti N, Batra YK, Jain A, Kumar P, Nikhar SA "The analgesic effect of intrathecal dexmedetomidine or clonidine, with bupivacaine, in trauma patients undergoing lower limb surgery: a randomised, double-blind study" Anaesth Intensive undergoing lower limb surgery: a randomised, double-blind study" Anaesth Intensive

Care. 201

4) Elia N, Culebras X, Mazza C, Schiffer E, Tramer MR. Clonidine as an adjuvant to intrathecal local anesthetics for surgery: Systematic review of randomized trials. Reg Anesth Pain Med 2008;33:159-67.

5) Grewal A. Dexmedetomidine: New avenues. J Anaesthesiol Clin Pharmacol 2011; 27:297-302.

6) Kanazi GE, Aouad MT, Jabbour-Khoury SI, Al Jazzar MD, Alameddine MM, AlYaman, et al. Effect of low-dose dexmedetomidine or clonidine on the characteristics of bupivacaine spinal block. Acta AnaesthesiolScand2006;50:222-7.

7) Katz J, Melzack R. Measurement of ain. Surg Clin North Am 1999·79:231-52.

7) Katz J, Melzack R. Measurement of pain. Surg Clin North Am 1999;79:231-52.
8) Al Ghanem SM, Massad IM, Al-Mustafa MM, Al-Zaben KR, Qudaisat IY, Qatawneh AM, et al. Effect of adding dexmedetomidine versus fentanyl to intrathecal bupivacaine on spinal block characteristics in gynecological procedures: A double blind controlled study. Am J Appl Sci 2009;6:882-7

9) Bajwa SJ, Bajwa SK, Kaur J, Singh G, Arora V, Gupta S, et al. Dexmedetomidine and clonidine in epidural anaesthesia: A comparative evaluation. Indian J Anaesth 2011; $55: 116-21$.

10) Al-Mustafa MM, Abu-Halaweh SA, Aloweidi AS, Murshidi MM, Ammari BA, AwwadZM, et al. Effect of dexmedetomidine added to spinal bupivacaine for urological procedures. Saudi Med J 2009;30:365-70. 